\title{
Análise bitemporal de áreas queimadas na Mata Atlântica
}

\author{
Bitemporal analysis of burnt areas in the Atlantic Forest
}

\section{Iorrana Figueiredo Sacramento 1 Roberto Ferreira Machado Michel ${ }^{2}$ Rafael Gomes Siqueira ${ }^{3}$}

\author{
Palavras-chave: \\ Classificação de imagem \\ Segmentação de imagem \\ Razão de bandas \\ Seleção de variáveis \\ Incêndios florestais
}

\begin{abstract}
Resumo
O estudo de áreas queimadas serve como subsídio para os planos de controle e monitoramento do fogo nas unidades de conservação. Diante dos desafios de caracterização do comportamento espectral de áreas queimadas, este estudo objetivou aplicar o método de classificação orientada a objeto e avaliar a melhor performance do uso conjunto de índices espectrais para o mapeamento de área queimadas na Mata Atlântica do Sul da Bahia. Para tanto, foi realizada análise bitemporal entre 2014 e 2016, considerando a diferença de cada índice espectral entre duas imagens LANDSAT 8: pré e pós-fogo. A classificação orientada a objeto foi executada de maneira supervisionada e automatizada por meio de algoritmos de segmentação, classificação e otimização em ambiente SIG. A classe de intensidade de queimada fraca foi a mais expressiva, com 13,65\% da área mapeada, enquanto a classe severa ocupou apenas $0,3 \%$. As áreas queimadas apresentaram um aumento da reflectância na faixa do vermelho e do infravermelho médio e uma diminuição na faixa do infravermelho próximo. $\mathrm{O} \triangle \mathrm{NBR}$ foi o melhor discriminador de áreas queimadas e o conjunto $\triangle \mathrm{NBR}, \triangle \mathrm{NBR} 2, \triangle \mathrm{NDMI}, \triangle \mathrm{SAVI}, \triangle \mathrm{NDVI}$, $\triangle$ GEMI e $\triangle$ MSAVI, apresentou o maior limiar de separação. A validação da classificação feita pelo coeficiente de concordância Kappa obteve um bom resultado (0,72). A seleção de variáveis mostrou-se eficiente na determinação do conjunto de índices espectrais de melhor performance para deteç̧ão das classes de queimadas, melhorando a acurácia da classificação e a confiabilidade dos resultados. A etapa de segmentação também se mostrou importante para a eficácia da classificação orientada a objeto, sendo influenciada diretamente pela resolução espacial da imagem.
\end{abstract}

Keywords:

Image classification

Image segmentation

Band ratio

Variables selection

Forest fire

\begin{abstract}
The study of burned areas is used as a subsidy for fire control and monitoring in the protected areas. In face of the challenges of the spectral signature characterization of burned areas, this study aimed to apply the object-oriented classification method and to evaluate the performance of spectral indices subsets for mapping burned areas in the Atlantic Forest. For that, we performed a bitemporal analysis between 2014 and 2016, considering the difference of each spectral indices among two LANDSAT 8 images: pre-and post-fire. The object-
\end{abstract}

${ }_{1}$ Programa de Pós-Graduação em Solos e Nutrição de Plantas - UFV. iorranafigueiredos@gmail.com

2 Departamento de Ciências Agrárias e Ambientais - UESC. roberto@michel.com

${ }^{3}$ Programa de Pós-Graduação em Solos e Nutrição de Plantas - UFV. rafael.geo.siqueira@gmail.com 
oriented classification was performed automatically by segmentation, supervised classification and optimization algorithms in the GIS environment. The "weak" burn severity class was the most expressive, with $13.65 \%$ of the mapped area, while the "severe" burn severity class occupied $0.3 \%$. The burned areas presented an increase of reflectance in the red and shortwave infrared bands and a decrease in the near infrared band. The $\triangle \mathrm{NBR}$ was the best discriminator of burned area and the $\triangle$ NBR, $\triangle$ NBR2, $\triangle$ NDMI, $\triangle$ SAVI, $\triangle$ NDVI, $\triangle \mathrm{GEMI}$ and $\triangle \mathrm{MSAVI}$ set presented the highest separation threshold. The validation of the classification by the Kappa agreement coefficient obtained a good outcome (0.72). The selection of the variables showed efficiency in determining the spectral indices' subset with the best performance for detecting the classes of burned areas, improving the classification accuracy and reliability. The segmentation was also important for the effectiveness of the object-oriented classification, being directly influenced by the image spatial resolution.

\section{INTRODUÇÃO}

A Mata Atlântica (MA) é um bioma historicamente ameaçado pela pressão antrópica, considerado um hotspot mundial que apresenta regiões bastante alteradas e fragmentadas (PINTO et al., 2006). Entre 1986 e 2001, apesar do aumento absoluto em 5\% da cobertura florestal na MA do Sul da Bahia, houve um intenso processo de fragmentação da paisagem, com aumento do número de fragmentos com menos de 100 hectares (SAVE BRASIL; IESC; BIRDLIFE INTERNATIONAL, 2009). Estudos na Reserva Biológica - REBIO de Una mostraram grande quantidade de cabrucas (plantações de cacau sombreadas pela floresta) e matas secundárias, que contribuem para a diminuição do isolamento e a permanência de populações de animais e plantas na região. Isto evidencia a importância da manutenção de áreas contíguas de Unidades de Conservação (UC).

As queimadas representam uma ameaça recorrente a áreas protegidas (SANTOS; SOARES; BATISTA, 2006; TORRES et al., 2017; 2018). O uso do fogo para o manejo do solo possui caráter milenar (BENTO-GOLÇALVES et al, 2012; CHUVIECO, 1997; MISTRY; BIZERRIL, 2011). O manejo tradicional é a principal estratégia agrícola no Refúgio de Vida Silvestre - REVIS de Una (SOLLBERG; SCHIAVETTI; MORAES, 2014) e, apesar desta técnica englobar práticas conservacionistas, também utiliza o corte e a queima. Os efeitos de uma queimada afetam a composição e estrutura das florestas, a biodiversidade animal, a saúde humana e o equilíbrio atmosférico, tanto em escala local quanto em escala regional
(COCHRANE, 2003; DODONOV et al., 2019; FREITAS et al., 2005; MENEZES; CAZETTA; DODONOV, 2019).

A criação de políticas de prevenção e monitoramento de incêndios tem se tornado possível através da aplicação de técnicas de Sensoriamento Remoto (SR), pois permitem extrair informações de forma rápida e eficiente, para serem utilizadas na identificação, controle e fiscalização de queimadas (CHUVIECO, 1996; DOS SANTOS et al., 2018; FREITAS et al., 2005; PEREIRA, 2009; SANTOS et al., 2020). A análise bitemporal de imagens de satélite tem sido uma técnica amplamente utilizada na deteç̧ão e monitoramento de mudanças ambientais, principalmente no que diz respeito à estimação de áreas queimadas e à deteç̧ão da severidade do fogo (CARDOZO et al., 2011; CARVAJAL-RAMIREZ et al., 2019; ESCUÍN; NAVARRO; FERNÁNDEZ, 2008; SANTOS et al. 2020). O método de classificação de imagens orientada a objetos tem se destacado por considerar não apenas a informação espectral, mas também relações espaciais como forma e contexto, resultando em classificações mais sofisticadas e próximas da interpretação humana, sem o efeito "salt and pepper" comum no método pixel a pixel (McDERMID, et al., 2003; WONG et al., 2003).

Entretanto, a deteç̧ão de áreas queimadas por SR tem apresentado limitações, devido à dificuldade de discriminação do fogo de possíveis falhas de imageamento, que podem muitas vezes limitar a deteç̧ão (COCHRANE, 2003). Além disso, a tendência de confusão entre superfícies queimadas e outros usos e coberturas do solo, tem levado à necessidade de uma melhor caracterização de sua assinatura espectral, o que tem sido possível por meio dos índices 
espectrais, que auxiliam na discriminação de alvos da superfície terrestre com base em seu comportamento espectral, por meio de cálculos aritméticos entre bandas (CARVAJALRAMIREZ et al., 2019; DOS SANTOS et al., 2018; LEITE et al., 2017; SANTOS et al., 2020). Dessa forma, os principais objetivos desta pesquisa foram aplicar o método de classificação orientada a objeto e avaliar a melhor performance do uso conjunto de índices espectrais para o mapeamento de áreas queimadas na Mata Atlântica do Sul da Bahia.

\section{MATERIAIS E MÉTODOS}

\section{Descrição da área de estudo}

A área de estudo $\left(709,7 \mathrm{~km}^{2}\right)$ compreende as UC de proteção integral: REBIO de Una $\left(187 \mathrm{~km}^{2}\right)$, REVIS de Una $\left(232 \mathrm{~km}^{2}\right)$ e suas zonas de amortecimento (3 mil metros no entorno), criadas em 1980 e 2007, respectivamente (BRASIL, 1980; 2007). A área abrange principalmente o município deUna, na região Sul da Bahia (Figura 1). Una possui população estimada de 19.000 habitantes e densidade demográfica de 16,86 hab./km² (IBGE, 2019). Sua economia é baseada principalmente na agricultura e no extrativismo, destacando-se a produção de cacau (cabrucas), borracha, café, mandioca, coco, açaí e banana (IBGE, 2017).

Figura 1 - Mapa de localização da REBIO de Una, REVIS de Una e a zona de amortecimento e da extensão da Mata Atlântica na área em 2018.

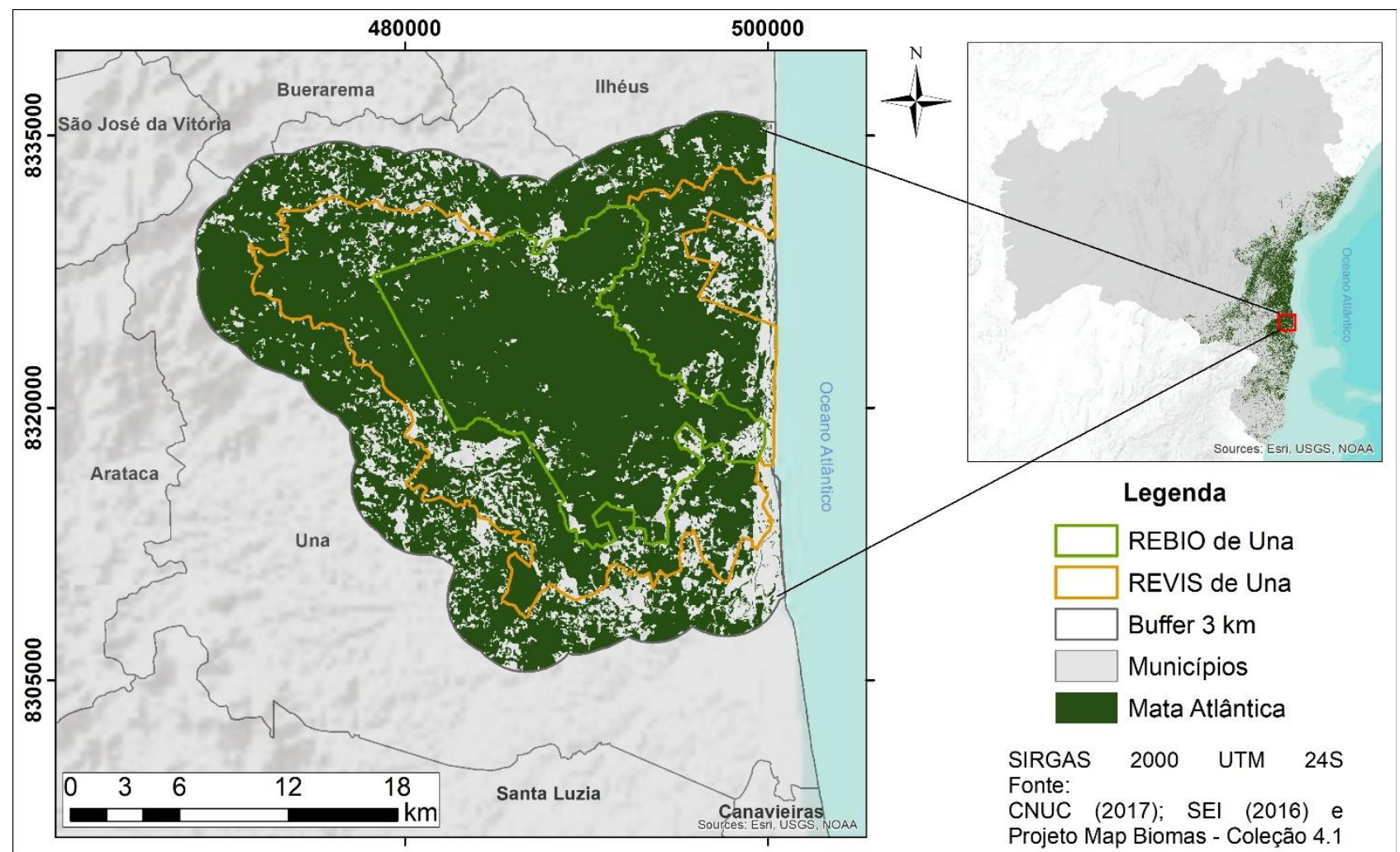

Fonte: MAPBIOMAS (2020). Org.: pelos autores, 2020.

A área de estudo integra o Complexo da Serra das Lontras, formado por 3 (três) serras interligadas que fazem parte da unidade geomorfológica das Serras e Maciços PréLitorâneos (SAVE BRASIL; IESB; BIRDLIFE INTERNATIONAL, 2009). A região é caracterizada por chuvas bem distribuídas durante o ano, com precipitações anuais em torno de $1.400 \mathrm{~mm}$ e temperatura média anual de $24,8{ }^{\circ} \mathrm{C}$ (Figura 2). A umidade relativa do ar registrada para 2014 foi de $84 \%$ (INMET, 2020).
Esta condição propicia a formação de solos profundos, bem drenados e porosos - Latossolos Amarelos e Vermelho-Amarelos - originados de rochas cristalinas (SAVE BRASIL; IESB; BIRDLIFE INTERNATIONAL, 2009). A Floresta Ombrófila Densa corresponde atualmente a $81 \%$ da área de estudo (Figura 1) e aumentou cerca de $1 \%$ desde 1988 (MAPBIOMAS, 2020), evidenciando a importância das UC para a conservação dos remanescentes da Mata Atlântica. 
Figura 2 - Média da temperatura média mensal e média da precipitação total mensal no período de 2012 e 2016 em Una - BA.

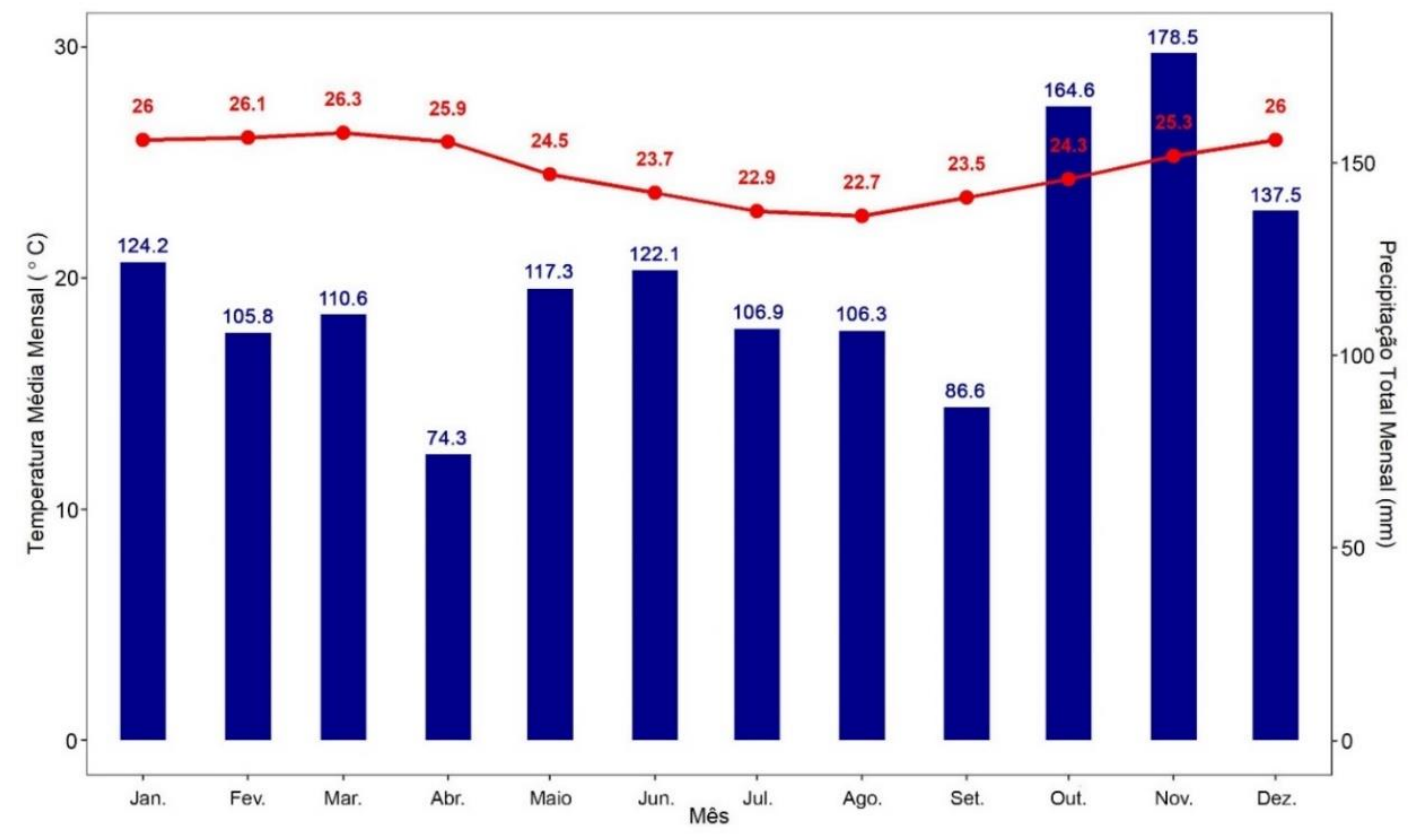

Fonte: INMET (2020). Org.: pelos autores, 2020.

\section{Materiais}

Foram utilizadas as bandas espectrais do sensor Operational Land Imager (OLI), satélite LANDSAT 8 (órbita-ponto 215-71), com resolução espacial de 30 metros (Tabela 1). Estes produtos foram obtidos pós-processados, com correção da geometria e reflectância da superfície - correção da absorção ou espalhamento da atmosfera (BARSI et al., 2014; VERMOTE et al., 2016). As bandas pancromáticas, de resolução espacial de 15 metros, foram utilizadas apenas na etapa de segmentação, com o objetivo de aumentar o detalhamento da mesma. A data da imagem préfogo é 07 de agosto de 2014 e a data da imagem pós-fogo é 06 de abril de 2016. Imagens de épocas distintas foram selecionadas devido a menor cobertura de nuvens e à ausência de períodos secos marcados na região (Figura 2), garantindo similar resposta espectral da vegetação ao longo do ano.

Os dados de ocorrência de focos de calor foram obtidos do Banco de Dados de Queimadas (BDQ) do Instituto Nacional de Pesquisas Espaciais (INPE, 2018), gerados por imagens dos satélites polares NOAA e EOS, e geoestacionários GOES e METEOSAT. Entre 2014 e 2016, foram identificados 224 focos na área de estudo. Essas informações serviram como evidência da ocorrência de fogo, sendo utilizadas na etapa de validação da classificação.

Tabela 1. Bandas espectrais do LANDSAT 8.

\begin{tabular}{ccc}
\hline Banda & Nomenclatura & Faixa (nm) \\
1 & Ultra blue & $435-451$ \\
2 & Azul (B) & $452-512,1$ \\
3 & Verde (G) & $532,7-5,90,1$ \\
4 & Vermelho (R) & $635,9-673,3$ \\
5 & Infravermelho próximo (NIR) & $850,5-878,8$ \\
6 & Infravermelho de banda curta (SWIR 1) & $1566,5-1651,2$ \\
7 & Infravermelho de banda curta (SWIR 2) & $2107,4-2294,1$ \\
8 & Pancromática (PAN) & $503,3-676,7$ \\
\hline
\end{tabular}

Fonte: USGS (2018). Org.: pelos autores, 2020. 


\section{Classificação orientada a objeto}

Todo o processamento foi realizado utilizando o software eCognition 9. Para a classificação, nuvens, sombras e corpos hídricos presentes nas imagens pré e pós fogo foram removidas. Com as imagens pós-fogo foram criadas 5 classes de detecção de mudanças da cobertura do solo: 1 severa, 2 - moderada, 3 - fraca (classes de intensidade de queimada); 4 - classe sem alteração; e 5 - classe de regeneração (mudança ocorrida pelo aumento da biomassa). $\mathrm{Na}$ definição das classes de queimadas, adotou-se o seguinte critério: a classe severa correspondeu ao epicentro de manchas de queimada observadas na imagem, a classe fraca às zonas de borda, enquanto a classe moderada à faixa de transição entre as duas zonas anteriores. Para cada classe, foram coletadas 15 amostras de treinamento, totalizando 75 amostras.

A segmentação foi realizada utilizando todas as bandas da imagem pós-fogo. $\mathrm{O}$ algoritmo utilizado foi o Multiresolution Segmentation (BAATZ; SHÄPE, 2000). Este algoritmo inicia a segmentação com cada pixel da imagem representando um objeto, o qual pode vir a se agrupar a um pixel vizinho, considerando parâmetros de escala e homogeneidade. O primeiro parâmetro define a heterogeneidade máxima permitida em um objeto e influencia no tamanho dos mesmos. $\mathrm{O}$ segundo diz respeito à relação entre cor e geometria dos objetos, para criação de objetos menos heterogêneos possível (DEFINIENS, 2012). Foram feitos testes para definição dos parâmetros descritos anteriormente, com valores de scale 80 , shape 0,2 e compactness 0,5 se mostrando mais adequados para a área de estudo.

Em seguida, foram calculados índices espectrais, por meio da razão de bandas, para as imagens pré-fogo e pós-fogo. Assim, calculou-se o: Índice de Vegetação da Diferença Normaliza NDVI (Equação 1) (ROUSE et al., 1973); Índice de Vegetação Melhorado - EVI (Equação 2) (JENSEN, 2009); Índice de Vegetação Ajustado ao Solo - SAVI (Equação 3) (HUETE, 1988); Índice de Vegetação Ajustado ao Solo Modificado - MSAVI (Equação 4) (QI et al., 1994); Índice de Umidade da Diferença Normalizada - NDMI (Equação 5) (JESEN, 2009); Índice de Queimada Normalizada - NBR (Equação 6) (KEY; BENSON, 1999); Índice de Queimada Normalizada 2 - NBR2 (Equação 7) (MARTÍN; GÓMEZ; CHUVIECO, 2005); Índice de Área Queimada - BAI (Equação 8) (CHUVIECO; MARTÍN; PALACIOS, 2002); Índice Global de Monitoramento Ambiental - GEMI (Equação 9a e 9b) (PINTY; VERSTRAETE, 1992).

$$
\begin{aligned}
& \mathrm{NDVI}=\frac{(\mathrm{NIR}-\mathrm{R})}{(\mathrm{NIR}+\mathrm{R})} \\
& \text { (Equação 1) } \\
& \mathrm{EVI}=\mathrm{G} *\left(\frac{(\mathrm{NIR}-\mathrm{R})}{(\mathrm{NIR}+\mathrm{C} 1 \mathrm{R}-\mathrm{C} 2 \mathrm{~B}+\mathrm{L})}\right) \quad \text {. (Equação 2) } \\
& \left.\mathrm{SAVI}=\left(\frac{(\mathrm{NIR}-\mathrm{R})}{(\mathrm{NIR}+\mathrm{R}+\mathrm{L})}\right) *(1+\mathrm{L}) \quad \text {.. (Equação } 3\right) \\
& \text { MSAVI }=\frac{\left.\left(2 \mathrm{NIR}+1-\sqrt{\left((2 \mathrm{NIR}+1) * 2-8^{*}(\mathrm{NIR}-\mathrm{R})\right)}\right)\right)}{2}(\text { Equação 4) } \\
& \mathrm{NDMI}=\frac{(\mathrm{NIR}-\mathrm{SWIR} 1)}{(\mathrm{NIR}+\mathrm{SWIR} 1)} \quad \text { (Equação 5) } \\
& \mathrm{NBR}=\frac{(\mathrm{NIR}-\mathrm{SWIR} 2)}{(\mathrm{NIR}+\mathrm{SWIR} 2)} \quad \text { (Equação 6) } \\
& \mathrm{NBR} 2=\frac{(\text { SWIR1-SWIR2) }}{(\text { SWIR1+SWIR2) }} \quad \text { (Equação 7) } \\
& \mathrm{BAI}=\frac{1}{\left.\left((0.1-\mathrm{R})^{2}\right)+\left((0.6-\mathrm{NIR})^{2}\right)\right)} \quad \text { (Equação 8) } \\
& \mathrm{GEMI}=\frac{\eta^{*}\left(1-0,25^{*} \eta\right)-(\text { RED-0,125) }}{(1-\mathrm{RED})} \ldots . . . . . \quad \text { (Equação 9a) }
\end{aligned}
$$

Em que:

$$
\left.\mathrm{\eta}=\frac{\left(2^{*}\left(\mathrm{NIR}^{2}-\mathrm{RED}^{2}\right)+1,5^{*} \mathrm{NIR}+0,5 * \mathrm{RED}\right)}{(\mathrm{NIR}+\mathrm{RED}+0,5)} \quad \text { (Equação } 9 \mathrm{~b}\right)
$$

Posteriormente, foram calculadas imagensdelta, que corresponderam à diferença entre o índice para a imagem pré e o mesmo índice para imagem pós ( $\Delta$ índice $=$ índice pré-fogo - índice pós-fogo). Como resultado da subtração dos índices pré-fogo - pós-fogo, as áreas das imagens delta onde a vegetação foi suprimida tiveram valores > 0 ; onde houve regeneração da vegetação, valores < 0; e onde não houve mudança, valores próximos de 0 (CARDOZO et al., 2011; PEREIRA, 2009).

Em seguida, foi utilizada a ferramenta Feature Space Optimization para definir quais imagens delta foram as mais adequadas para a classificação. Esta ferramenta ranqueia as variáveis (imagens delta) por ordem de importância e cria um número de sub-grupos (dimensões) de diferentes tamanhos, proporcional ao número de variáveis de entrada. Cada dimensão engloba um número de variáveis diferentes, de forma cumulativa ( $\operatorname{dim} 1=$ uma variável; $\operatorname{dim} 2=$ duas variáveis, $\operatorname{dim} 3=$ três variáveis... $\operatorname{dim} n=n$ variáveis). Para cada 
dimensão, o algoritmo calcula a distância Euclidiana entre os objetos de cada classe a ser mapeada, a fim de encontrar a combinação de variáveis que apresenta a maior distância (dada entre 0 e 1) e consequentemente o maior limiar de separação das classes (TRIMBLE, 2014).

A partir disto, a classificação supervisionada foi realizada através do algoritmo Nearest Neighbor (Vizinho Mais Próximo). Este algoritmo funciona considerando a distância a qual o objeto a ser classificado se encontra da amostra de treinamento mais próxima, isto é, quanto mais próximo for um objeto de uma amostra, maior será o grau de relação entre eles (GABRIEL, 2013) e, assim, ele será agrupado à classe dessa amostra. O Nearest Neighbor foi o algoritmo de melhor performance entre os classificadores orientados a objeto testados por Zanotta et al. (2010).

Para a validação da classificação, foi aplicado o cálculo do índice Kappa, considerado em muitos estudos como apropriado para aferir a acurácia de uma classificação (GABRIEL, 2013). Este índice foi aplicado a partir da ferramenta Accuracy Assestment e foram utilizadas 65 amostras, coletadas com o auxílio os focos de calor do INPE. Segundo Landis e Koch (1977), os valores do índice Kappa podem variar de 0 a 1 , sendo $0-0,2=$ ruim; $0,2-0,4=$ razoável; $0,4-$ $0,6=$ bom; $0,6-0,8=$ muito bom; e $0,8-1,0=$ excelente. Resumidamente, todo o procedimento metodológico está apresentado abaixo (Figura $3)$.

Figura 3 - Etapas do método de classificação orientada a objeto.

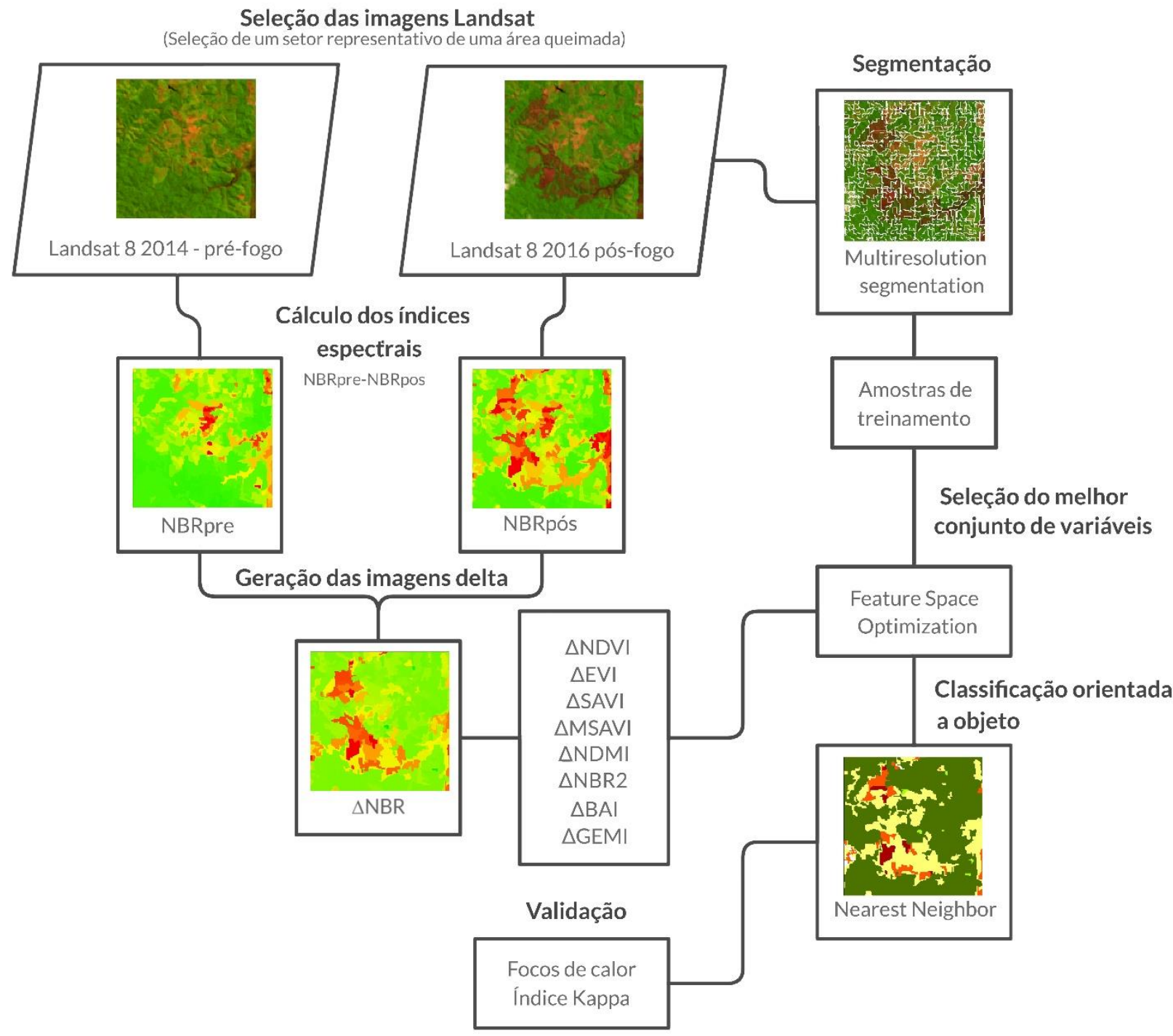

Fonte: Os autores, 2020. 


\section{RESULTADOS E DISCUSSÃO}

A figura 4a apresenta os valores médios de reflectância de uma amostra-padrão de área queimada para as bandas espectrais das imagens pré e pós-fogo. Observa-se que houve um aumento da reflectância na região do visível (RGB) e do infravermelho médio (SWIR1 e SWIR2), com maior alteração no visível ocorrendo na faixa do vermelho (R). Na imagem pré-fogo, em condição de maior biomassa, a reflectância da região do vermelho $(\mathrm{R})$ foi menor que o da região do verde $(G)$, situação invertida na imagem pós-fogo.
Por sua vez, a faixa do infravermelho próximo (NIR) sofreu uma significativa diminuição da reflectância (MARTÍN; CHUVIECO; 1998). Isto provocou uma diminuição na amplitude de reflectância entre as bandas NIR e R, e NIR e SWIR, o que afetou diretamente o comportamento dos índices espectrais. A figura $4 \mathrm{~b}$ apresenta os valores de reflectância dos pixels de áreas queimadas para as bandas R, NIR e SWIR2. Os valores de reflectância das áreas queimadas nas bandas $R$ e SWIR2 foram mais altos na imagem pós fogo, enquanto os valores na banda NIR foram maiores na imagem pré-fogo.

Figura 4 - a) Média da reflectância de objetos de áreas queimadas nas bandas das imagens pré e pós-fogo; b) Reflectância dos pixels de áreas queimadas para as bandas R, NIR e SWIR2.
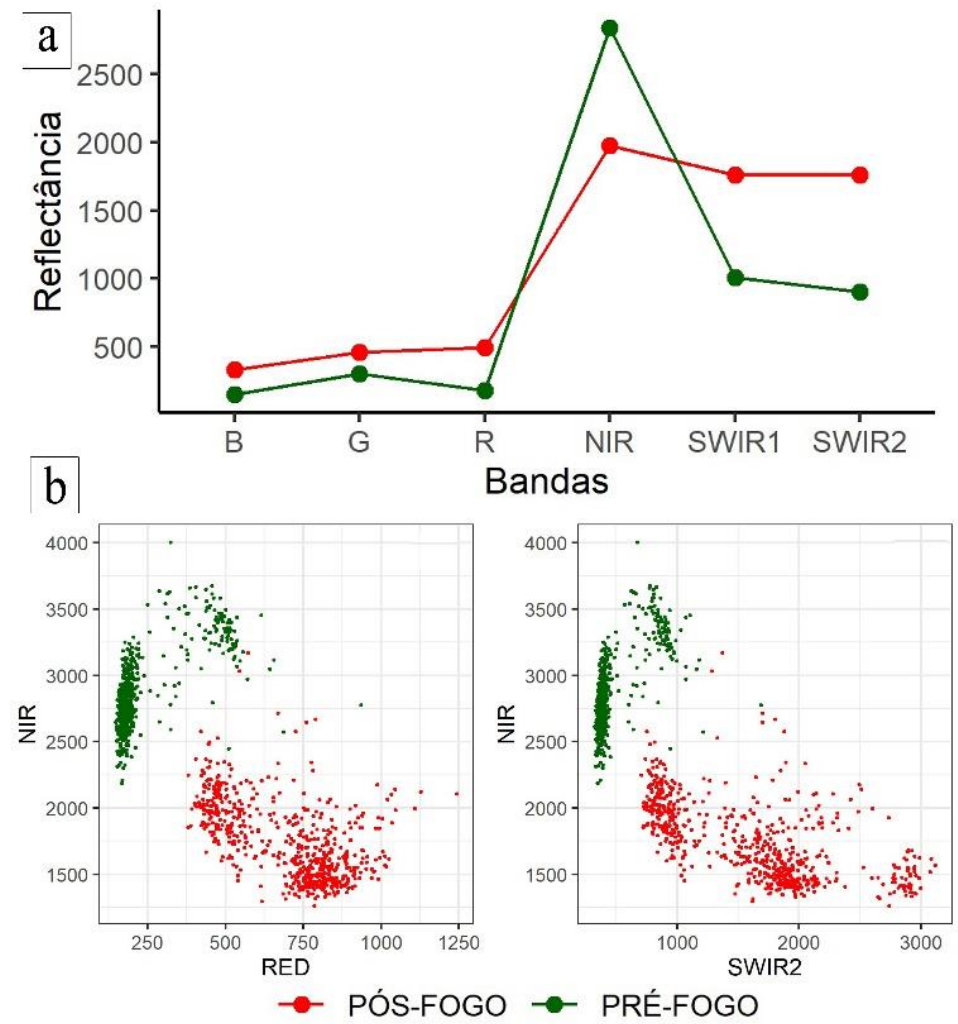

Fonte: Os autores, 2020.

O aumento da reflectância no visível, bem como o aumento da reflectância da faixa $\mathrm{R}$ em relação à $\mathrm{G}$ (em cerca de $8 \%$ ), é explicado pela redução da absorção da radiação pelos pigmentos fotossintetizantes, devido à perda de biomassa causada pela queima (PONZONI; SHIMABUKURO, 2007). Apesar dessa diferença, sabe-se que a região do visível não é a melhor indicada para a discriminação de áreas queimadas, devido à sua baixa reflectância e influência do espalhamento da atmosfera, principalmente sobre superfícies escuras, como áreas queimadas, o que diminui ainda mais o contraste entre os alvos terrestres (PEREIRA et al., 1999).

O decréscimo de $30,5 \%$ da reflectância no NIR pode ser explicado pela destruição da estrutura celular da planta, provocada pela queima. Devido à grande amplitude de reflectância desta faixa nas imagens pré e pósfogo, o NIR foi destacado como a melhor faixa do espectro para a discriminação de áreas queimadas (PEREIRA et al., 1999). O significativo aumento dos valores de reflectância 
para as faixas do SWIR1 e SWIR2 (75,4\% e $94,7 \%$, respectivamente) é devido à diminuição do conteúdo de água presente na folha (MARTÍN; CHUVIECO, 1998). A faixa do infravermelho médio foi reconhecida como efetiva na distinção de queimadas, devido à sua alta reflectância e pela insignificante influência do espalhamento atmosférico nessa faixa (PEREIRA et al., 1999).

O algoritmo Feature Space Optimization foi eficiente na seleção do conjunto de imagens delta mais adequado para a classificação (Figura 5 e Tabela 2). Considerando apenas um índice para análise $\left(1^{\mathrm{a}}\right.$ dimensão), o $\Delta \operatorname{NBR}(0,215)$ foi a imagem delta que possuiu maior capacidade de discriminação das classes de detecção de mudanças. Entretanto, o maior limiar de separação $(0,564)$ foi encontrado quando considerados sete dos nove índices calculados $\left(7^{\mathrm{a}}\right.$ dimensão). A partir da sétima dimensão, o alto número de imagens delta induziu redundâncias que diminuíram o limiar de separação das classes. A eficiência do algoritmo Feature Space Optmization já foi destacada em estudos anteriores para o aumento da performance da classificação de imagens (DURRIEU et al., 2007; LEDUC, 2004).

Figura 5 - Representação dos limiares de separação de cada dimensão analisada.

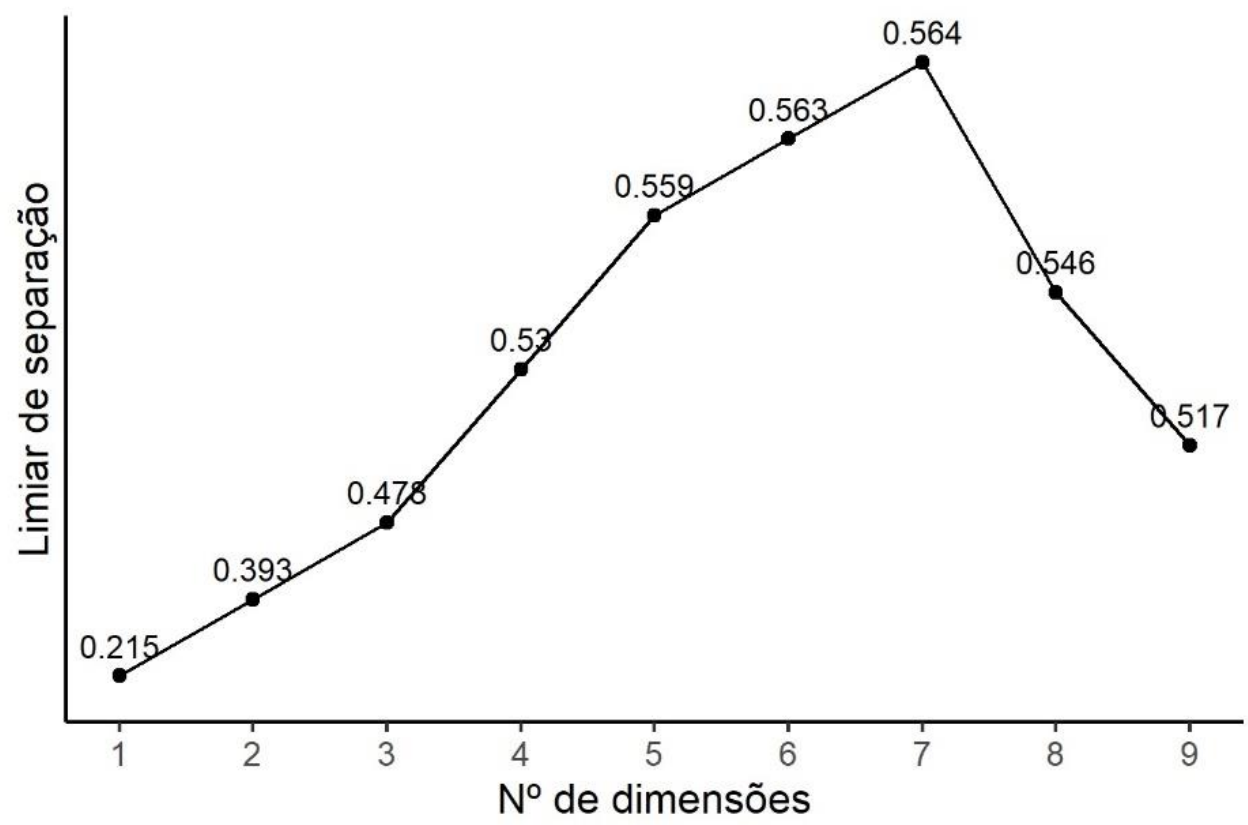

Fonte: Os autores, 2020.

Tabela 2. Dimensões de análise e seus respectivos limiares de separação.

\begin{tabular}{|c|c|c|}
\hline Dimensão & Imagem Delta & Limiar \\
\hline $1^{\mathrm{a}}$ & $\triangle \mathrm{NBR}$ & 0,215 \\
\hline $2^{\mathrm{a}}$ & $\triangle \mathrm{NBR}$ e $\triangle \mathrm{NBR} 2$ & 0,393 \\
\hline $3^{\mathrm{a}}$ & $\triangle \mathrm{NBR}, \triangle \mathrm{NBR} 2$ e $\triangle \mathrm{NDMI}$ & 0,478 \\
\hline $4^{\mathrm{a}}$ & $\Delta \mathrm{NBR}, \Delta \mathrm{NBR} 2, \Delta \mathrm{NDMI}$ e $\Delta \mathrm{SAVI}$ & 0,530 \\
\hline $5^{\mathrm{a}}$ & $\Delta \mathrm{NBR}, \Delta \mathrm{NBR} 2, \Delta \mathrm{NDMI}, \Delta \mathrm{SAVI}$ e $\Delta \mathrm{NDVI}$ & 0,559 \\
\hline $6^{\mathrm{a}}$ & $\Delta \mathrm{NBR}, \Delta \mathrm{NBR} 2, \Delta \mathrm{NDMI}, \Delta \mathrm{SAVI}, \Delta \mathrm{NDVI}$ e $\Delta \mathrm{GEMI}$ & 0,563 \\
\hline $7^{\mathrm{a}}$ & $\triangle \mathrm{NBR}, \Delta \mathrm{NBR} 2, \Delta \mathrm{NDMI}, \Delta \mathrm{SAVI}, \Delta \mathrm{NDVI}, \Delta \mathrm{GEMI}$ e $\triangle \mathrm{MSAVI}$ & 0,564 \\
\hline $8^{\mathrm{a}}$ & $\Delta \mathrm{NBR}, \Delta \mathrm{NBR} 2, \Delta \mathrm{NDMI}, \Delta \mathrm{SAVI}, \Delta \mathrm{NDVI}, \Delta \mathrm{GEMI}, \Delta \mathrm{MSAVI}$ e $\Delta \mathrm{BAI}$ & 0,546 \\
\hline $9^{\mathrm{a}}$ & $\Delta \mathrm{NBR}, \Delta \mathrm{NBR} 2, \Delta \mathrm{NDMI}, \Delta \mathrm{SAVI}, \Delta \mathrm{NDVI}, \Delta \mathrm{GEMI}, \Delta \mathrm{MSAVI}, \Delta \mathrm{BAI}$ e $\Delta \mathrm{EVI}$ & 0,517 \\
\hline
\end{tabular}

Fonte: Os autores, 2020.

Os índices de queimada NBR e NBR2 foram os mais significativos neste trabalho e os que melhor discriminaram áreas queimadas de acordo trabalhos anteriores (CARDOZO et al., 2011; ESCUÍN; NAVARRO; FERNÁNDEZ,
2008; PEREIRA, et al., 2015; ROSAN; ALCÂNTARA, 2015). Isto se dá pelo fato das bandas utilizadas em seus cálculos (NIR e SWIR2) serem as que sofreram as alterações mais significativas no pós-fogo. O $\triangle$ NDMI foi a 
terceira melhor imagem para discriminação das classes de queimada. O índice de umidade NDMI também apresentou maior potencial para detecção de queimadas do que índices de vegetação em Wilson e Sader (2002), o que é devido às mudanças no regime de umidade do solo e das plantas após a queima.

Dentre os índices de vegetação, o SAVI teve melhor capacidade de distinção das queimadas, tendo também apresentado performance moderada por Pereira, et al., (2015). O MSAVI é similar ao SAVI, porém este último utiliza um fator de correção do efeito do solo ajustado manualmente, de acordo com as condições ambientais de cada área, enquanto o MSAVI utiliza um fator auto-ajustado (QI et al. 1994), cuja rigidez pode induzir à resultados inferiores ao SAVI, como aconteceu neste trabalho. O NDVI, apesar de amplamente aplicado, não apresenta grande eficácia na detecção de áreas queimadas (CARDOZO et al., 2011; ESCUÍN; NAVARRO; FERNÁNDEZ, 2008; PEREIRA, 1999; PEREIRA, et al., 2015). O GEMI provou ser um índice melhor que o NDVI para a detecção de queimadas, por ser menos afetado pela influência atmosférica e do solo (PEREIRA, 1999). Aqui, a diferença da performance das imagens delta do NDVI e do GEMI não foi significativa, com ambos os índices possuindo uma performance moderada. O BAI não contribuiu para o aumento da separabilidade das classes, apesar de considerado um bom índice para a detecção de queimadas (CHUVIECO; MARTÍN, PALACIOS, 2002; DEMPWOLF, et al., 2007). O EVI, assim como o BAI, não contribuiu para o aumento da diferenciação de áreas queimadas, tendo também apresentado baixa separabilidade segundo Pereira et al. (2015).

Segundo a matriz de distância Euclidiana entre as classes de detecção de mudanças (Tabela 3), as classes sem alteração e regeneração apresentaram menor valor de separação $(0,56)$, uma vez que se assemelharam em termos espectrais por ambas corresponderem a áreas de vegetação. A classe de queimada severa apresentou os maiores valores de separabilidade com as classes anteriores (28,6 e 37,7), devido à grande perda de biomassa associada à queima. Entre as classes de intensidade de queimada (classes 1, 2 e 3), as classes severa e moderada apresentaram menor valor de separação $(0,9)$ e as classes severa e fraca apresentaram o maior $(4,7)$. Comparando as distâncias entre as classes de queimada e as demais, percebe-se que o menor valor de separação foi entre as classes fraca e sem alteração $(5,3)$.

Tabela 3. Matriz de distância Euclidiana apresentando a separação por classe: 1 - severa; 2 moderada; 3 - fraca; 4 - sem alteração; e 5 - regeneração.

\begin{tabular}{cccccc}
\hline Classes & $\mathbf{1}$ & $\mathbf{2}$ & $\mathbf{3}$ & $\mathbf{4}$ & $\mathbf{5}$ \\
1 & 0 & 0,9 & 4,7 & 28,6 & 37,7 \\
2 & 0,9 & 0 & 1,4 & 17,3 & 24,6 \\
3 & 4,7 & 1,4 & 0 & 5,3 & 9,4 \\
4 & 28,6 & 17,3 & 5,3 & 0 & 0,6 \\
5 & 37,7 & 24,6 & 9,4 & 0,6 & 0 \\
\hline
\end{tabular}

Fonte: Os autores, 2020.

A classe de intensidade fraca corresponde principalmente às zonas de borda das áreas queimadas, onde a diferença espectral entre as imagens pré e pós-fogo foi menor, enquanto a classe severa corresponde ao epicentro da ocorrência das queimadas, onde as mudanças espectrais induzidas pelo fogo foram maiores. A semelhança entre os valores de distância da classe fraca com as classes severa e sem alteração indica a condição de transição da classe de queimada fraca na área de estudo.

De acordo com a classificação final, observa- se que cerca de $74 \%$ da área total não passou por mudanças significativas na cobertura vegetal no período analisado (Figura 6; Tabela 4). As classes de áreas queimadas representaram $16,1 \%$ da área total mapeada e foram mais pronunciadas na região nordeste correspondente à parte da REVIS de Una e da zona de amortecimento (Figura 6), áreas em que o uso é permitido e mais intensificado. Dessa porcentagem, 13,65\% corresponderam à classe fraca, enquanto que apenas $0,3 \%$ correspondeu à classe severa (Tabela 4). 
Figura 6 - Classificação orientada a objeto da área de estudo.

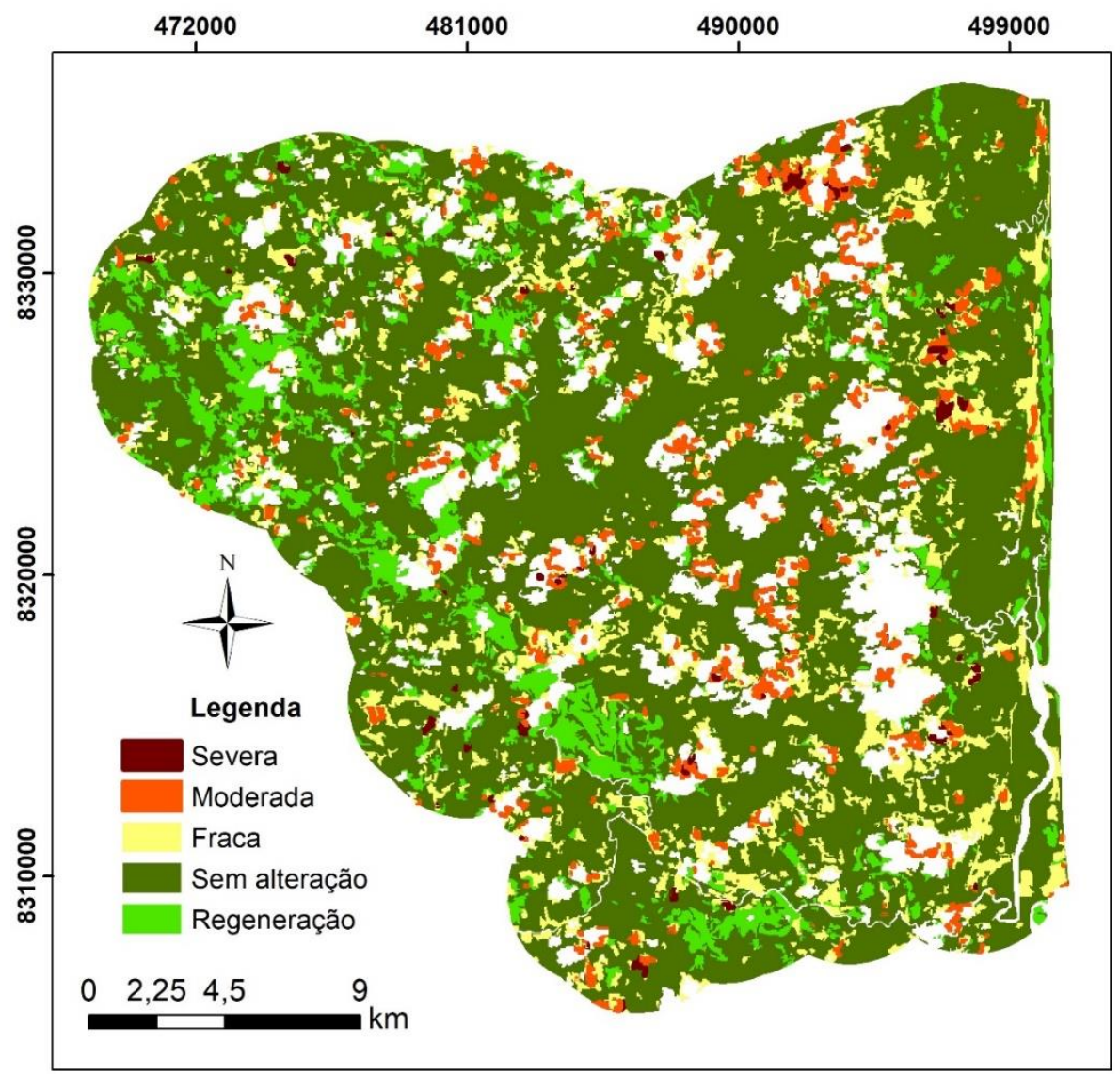

Fonte: Os autores, 2020.

No entanto, observou-se também uma certa superestimação da classe fraca. Tal superestimação pode ter ocorrido por três razões: em áreas onde a mudança foi pouco percebida, com valores positivos próximos de 0 nas imagens delta, como nos cordões litorâneos; áreas ao redor das nuvens, cuja influência resultou em valores positivos próximos de 1 ; ou a confusão gerada pela presença de pixels estranhos no interior de alguns objetos. Isto pode estar associado à baixa resolução espacial da imagem LANDSAT8, interferindo no processo de segmentação, uma vez que pixels não queimados foram incluídos em alguns objetos das classes de queimada, principalmente a classe fraca.

Do total de áreas de queimadas mapeadas $\left(95.23 \mathrm{~km}^{2}\right), 45 \%$ foram identificadas na zona de amortecimento; $34 \%$ na REVIS de Una; e $21 \%$ na REBIO de Una, indicando a maior efetividade desta última UC no combate e prevenção ao fogo. O valor Kappa para a classificação foi de 0,72 , o que de acordo com Landis e Koch (1977) corresponde à uma classificação de nível muito bom $(0,6-0,8)$.

Tabela 4 - Quantificação das classes de área queimada e vegetação da área de estudo.

\begin{tabular}{ccc} 
Classe & Área $\mathbf{( k m}^{\mathbf{2}} \mathbf{)}$ & Área (\%) \\
Severa & 1,9 & 0,3 \\
Moderada & 12,7 & 2,15 \\
Fraca & 80,7 & 13,65 \\
Sem alteração & 436,5 & 73,82 \\
Regeneração & 59,6 & 10,08 \\
Total & 591,3 & 100 \\
\hline
\end{tabular}

Fonte: Os autores, 2020. 


\section{CONCLUSÃO}

O uso de imagens delta de índices espectrais pré e pós-fogo mostrou-se eficiente na detecção de mudanças na cobertura do solo através de queimadas na Mata Atlântica do sul da Bahia. Com a seleção de variáveis através do Feature Space Optimization foi possível identificar os índices espectrais mais importantes para a deteç̧ão de tais mudanças, bem como identificar o subconjunto de índices mais adequado para o aumento da acurácia da classificação de queimadas orientada a objeto. A imagem delta do índice de área queimada NBR foi a que apresentou melhor performance, enquanto o subconjunto com sete índices espectrais apresentou o maior limiar de separação, envolvendo a combinação de índices de queimada, de umidade e de vegetação.

Nossos resultados mostraram que o uso integrado dos diferentes índices espectrais potencializa a performance do classificador quando comparado ao uso particular de qualquer um dos índices. No entanto, observa-se que o aumento da performance não é diretamente proporcional ao aumento de variáveis, uma vez que o uso de mais de sete índices trouxe um aumento da confusão e redundância de informações, o que contribuiu para diminuir o grau de separabilidade entre as classes. Nesse sentido, a seleção de variáveis torna-se crucial para a garantia da acurácia da classificação e da confiabilidade dos resultados.

A classificação orientada a objeto também se mostrou fortemente dependente da etapa de segmentação, uma vez que a classificação é aplicada diretamente sobre os objetos criados na segmentação, tendo os mesmos que englobar de forma mais precisa possível pixels que correspondem ao mesmo alvo terrestre. A resolução média a baixa das imagens LANDSAT8 induziram certa confusão espectral dentre as classes avaliadas. Uma alternativa para a obtenção de objetos mais homogêneos em imagens de resolução espacial limitada seria o uso do método de mistura espectral, que possibilita a identificação da influência da resposta de diversos possíveis alvos (vegetação, solo e sombra) em um pixel de área queimada.

\section{REFERÊNCIAS}

BARSI, J. A. et al. The spectral response of the Landsat-8 Operational Land Imager, Remote Sensing, v. 6, p. 10232-10251, 2014. https://doi.org/10.3390/rs61010232

BAATZ, M.; SCHÄPE, A. Multiresolution segmentation: an optimization approach for high quality multi-scale image segmentation. In: STROBL; BLASCHLE; GRIESEBNER (Org.) Angewandte Geographische Informationsverarbeitung XII, Heidelberg: Wichmann-Verlag, 2000.

BENTO-GONÇALVES, A. et al. Fire and soils: Key concepts and recent advances. Geoderma, v. 191, p. 3-13, 2012. https://doi.org/10.1016/j.geoderma.2012.01.004

BRASIL. Decreto Federal n. 85.463, de 10 de dezembro de 1980. Disponível em: < http://www.planalto.gov.br/ccivil_03/Atos/decret os/1980/D85463.html>. Acesso em: 13 de agosto de 2020.

BRASIL. Decreto Federal s/n., de 21 de dezembro de 2007. Disponível em: <http://www.planalto.gov.br/ccivil_03/_ato20072010/2007/dnn/Dnn11459.htm>. Acesso em: 13 de agosto de 2020.

CARDOZO, F. S. et al. Avaliação de áreas queimadas a partir dos índices espectrais NDVI e NDBR. In: Simpósio Brasileiro de Sensoriamento Remoto, 15., 2011, Anais... Curitiba, 2011. p. 7950-7957. Disponível em: <http://marte.sid.inpe.br/col/dpi.inpe.br/marte/2 011/07.27.21.58/doc/p1429.pdf>. Acesso em: $10 \mathrm{de}$ abril de 2018.

CARVAJAL-RAMIREZ, F. et al. Evaluation of Fire Severity Indices Based on Pre- and Post-Fire Multispectral Imagery sensed from UAV. Remote Sensing, v. 11, n. 993, p. 1-19, 2019. https://doi.org/10.3390/rs11090993

CHUVIECO, E. (Ed.). A review of remote sensing methods for the study of large wildland fires. Spain: Universidad de Alcalá, 1997. $192 \mathrm{p}$.

CHUVIECO, E. Empleo de imágenes de satélite para medir la estrutura del paisaje: análisis cuantitativo y representación cartográfica. Serie Geográfica, v. 6, p. 131-147, 1996. Disponível em:

<https://core.ac.uk/download/pdf/58902321.pdf>. Acesso: 20 de abril de 2018.

CHUVIECO, E.; MARTÍN, M. P.; PALACIOS, A. Assessment of different spectral indices in the red-near-infrared spectral domain for burned land discrimination. International Journal of Remote Sensing, v. 23, n. 23, p. 5103-5110. 2002.

https://doi.org/10.1080/01431160210153129

COCHRANE, M. A. Fire science for rainforests. Nature, v. $421, \quad$ p. $913-919, \quad 2003$. https://doi.org/10.1038/nature01437

DODONOV, P. Air and soil temperature across fire-created edges in a Neotropical rainforest. Agricultural and Forest Meteorology, v. 276277 , 2019. https://doi.org/10.1016/j.agrformet.2019.06.005 
DOS SANTOS, J. F. C., et al. Potentials and limitations of remote fire monitoring in protected areas. Science of the Total Environment, v. 616-617, p. 1347-1355, 2018. https://doi.org/10.1016/j.scitotenv.2017.10.182

DURRIEU, S. et al. Influence of training sampling protocol and of feature space optimization methods on supervised classification results. 2007. IEEE International Geoscience and Remote Sensing Symposium, Barcelona, p. 2030-2033, 2007. https://doi.org/10.1109/IGARSS.2007.4423229

DEFINIENS. Developer XD 2.0.4: reference book. Munique: DEFINIENS, 2012, $414 \mathrm{p}$.

DEMPWOLF, J., et al. Burned-Area mapping of the serengeti-mara region using MODIS reflectance data. IEEE Geoscience and Remote Sensing Letters, v. 4, n. 2, p. 312-316, 2007. https://doi.org/10.1109/LGRS.2007.894140

ESCUÍN, S.; NAVARRO, R.; FERNÁNDEZ, P. Fire severity assessment by using NBR (Normalized Burn Ratio) and NDVI (Normalized Difference Vegetation Index) derived from LANDSAT TM/ETM images. International Journal of Remote Sensing, v. 29, n. 4, p. 1053-1073, 2008. https://doi.org/10.1080/01431160701281072

FREITAS, et al. Emissões de queimadas em ecossistemas da América do Sul. Estudos Avançados, vol. 19, n. 53, p. 167-185, 2005. https://doi.org/10.1590/S0103-

40142005000100011

GABRIEL, C. G. F. Análise comparada de segmentação e classificação orientada por objectos de uma imagem Worldview-2. 2013. 153 f. Dissertação (Mestrado em Gestão do território) - Universidade Nova de Lisboa, Lisboa, 2013.

HUETE, A. R., A soil-adjusted vegetation index (SAVI). Remote Sensing of Environment, vol. 25, p. $\quad 295-309$. 1988 https://doi.org/10.1016/0034-4257(88)90106-X

IBGE. Censo Agropecuário. 2017. Disponível em:

<https://cidades.ibge.gov.br/brasil/ba/una/pesqui sa/24/76693>. Acesso em: 26 de maio de 2020.

IBGE. Estimativa da população - 2019. Disponível em: <https://cidades.ibge.gov.br/brasil/ba/una/panora ma>. Acesso em: 26 de maio de 2020.

INMET. BDMEP - Banco de dados meteorológicos para ensino e pesquisa. Disponível em: < http://www.inmet.gov.br/portal/index.php?r=bd mep/bdmep>. Acesso em: 26 de maio de 2020.

INPE - Instituto Nacional de Pesquisas Espaciais. Banco de dados de queimadas. 2018. Disponível em:

$<$ http://queimadas.dgi.inpe.br/queimadas/bdq ueimadas>. Acesso em: 22 de março 2018.
JENSEN, J.R. Sensoriamento remoto do ambiente: uma perspectiva em recursos terrestres. São José dos Campos: Parêntese, 2009. p 357-410

KEY, C. H.; BENSON, N. C. The Normalized Burn Ratio (NBR): A Landsat TM radiometric measure of burn severity. US Dept. Interior, Northern Rocky Mountain Sci. Center, Bozeman, MT. 1999.

LANDIS, J.R.; KOCH, G.G. The measurement of observer agreement for categorical data. Biometrics, vol. 33, n. 1, 1997. pp. 159-174. https://doi.org/10.2307/2529310

LEDUC, F. Feature space optimization prior to fuzzy image classification. In: Proceedings of the $7^{\text {th }}$ International Conference of Information Fusion, p. 547-554, 2004. Disponível em: <http://www.fusion2004.foi.se/papers/IF040547.pdf>. Acesso em: 15 setembro de 2018.

LEITE, C. C. S. de S., et al. Análise dos incêndios ocorridos no Parque Nacional da Chapada Diamantina-Bahia em 2008 e 2015 com suporte em índices espectrais de vegetação. Revista Brasileira de Cartografia, v. 69, n. 6, p. 1127 1141, 2017. Disponível em: <http://www.seer.ufu.br/index.php/revistabrasile iracartografia/article/view/44315/23397>. Acesso em: 26 de maio de 2020.

MAPBIOMAS. Coleção 4.1 da Série Anual de Mapas de Cobertura e Uso de Solo do Brasil. 2020. Disponível em: <https://mapbiomas.org/>. Acesso em 28 de maio de 2020.

MARTÍN, M. P.; GÓMEZ, I.; CHUVIECO, E. Performance of a burned-area index (BAIM) for mapping Mediterranean burned scars from MODIS data. In: RIVA, J.; PÉREZ-CABELLO, F.; CHUVIECO, E. (Eds.). Proceedings of the 5th International Workshop on Remote Sensing and GIS applications to Forest Fire Management: Fire Effects Assessment, Paris, p. 193-198, 2005.

MARTÍN, M. P. I.; CHUVIECO, E. Cartografía de grandes incêndios forestales en la península ibérica a partir de imágenes NOAA-AVHRR. Serie Geográfica, vol. 7, p. 109-128, 1998. Disponível <https://digital.csic.es/handle/10261/6426>. Acesso em: 20 de abril de 2018.

McDERMID, G.P. et al. Object-oriented analysis for change detection. In: Proceedings of the $25^{\text {th }}$ Canadiam symposium on remote sensing, 14-17 October 2003, Montreal, Canada. Disponível em: $<$ https://www.researchgate.net/publication/2378 10865_OBJECT_ORIENTED_ANALYSIS_FOR_ CHANGE_DETECTION>. Acesso em: 20 junho de 2018.

MENEZES, G. S. C.; CAZETTA, E.; DODONOV, P. Vegetation structure across fire edges in a Neotropical rain forest. Forest Ecology and 
$\begin{array}{llll}\text { Management, } & \text { v. } & 453, & \end{array}$ https://doi.org/10.1016/j.foreco.2019.117587

MISTRY, K. BIZERRIL, M. Por que é importante entender as inter-relações entre pessoas, fogo e áreas protegidas? Biodiversidade Brasileira, vol. 1, n. 2, p. 40-49, 2011. Disponível em: <https://www.icmbio.gov.br/revistaeletronica/ind ex.php/BioBR/article/view/137>. Acesso: 20 de março de 2018.

PEREIRA, A. A. et al. Avaliação de nove índices espectrais quanto a separabilidade entre queimadas e diferentes alvos. In: Simpósio Brasileiro de Sensoriamento Remoto, 17. Anais... João Pessoa, p. 3105-3112, 2015.

PEREIRA, A. A. Uso de geotecnologia para detecção e análise de queimadas e focos de calor em unidades de conservação no norte de Minas Gerais. 2009. 91 f. Dissertação (Mestrado em Engenharia Florestal) Universidade Federal de Lavras, Lavras, 2009.

PEREIRA, J. M. C. A Comparative Evaluation of NOAA/AVHRR Vegetation Indexes for Burned Surface Detection and Mapping. IEEE Transactions on Geoscience and Remote $\begin{array}{lllll}\text { Sensing, } & \text { v. } 37, \quad \text { n. } & 1, & 1999 .\end{array}$ https://doi.org/10.1109/36.739156

PINTO, L. P. et al. Mata Atlântica Brasileira: os desafios para conservação da biodiversidade de um hotspot mundial. In: ROCHA, C. F. D. et al. (Ed.). Biologia da Conservação: essências. São Carlos: RiMa, p. 69-96, 2006.

PINTY, B.; VERSTRAETE, M. M., GEMI: A nonlinear index to monitor global vegetation from satellites, Vegetation, v. 101, n. 1, p. 15-20, 1992. https://doi.org/10.1007/BF00031911

PONZONI, F. J.; SHIMABUKURO, Y. E. Sensoriamento remoto no estudo da vegetação. São José dos Campos: Parêntese, 2007. 127 p.

QI, J. et al., Modified Soil Adjusted Vegetation Index (MSAVI). Remote Sensing of Environent, v. 48, n. 2, p. 119-126, 1994. https://doi.org/10.1016/0034-4257(94)90134-1

ROSAN, T. M.; ALCÂNTARA, E. Detecção de áreas queimadas e severidade a partir do índice espectral $\triangle$ NBR. In: In: Simpósio Brasileiro de Sensoriamento Remoto, 17. Anais... João Pessoa, 2015. p. 526-533.

ROUSE, J.W. et al. Monitoring vegetation systems in the Great Plains. Earth Resources Tecnology Satellite-1 Symposium, Washington, DC, p. 309-317, 1973.

SANTOS, J. F.; SOARES, R. V.; BATISTA, A. C. Perfil dos incêndios florestais no Brasil em áreas protegidas no período de 1998 a 2002. Floresta, v. $36, \quad$ n. $\quad 1 . \quad 2006$. https://doi.org/10.5380/rf.v36i1.5510

SANTOS, S. M. B. dos, et al. Assessment of Burned Forest Area Severity and Postfire Regrowth in Chapada Diamantina National Park (Bahia, Brazil) Using dNBR and RdNBR Spectral
Indices. Geosciences, v.10, n. 106, 2020. https://doi.org/10.3390/geosciences10030106
SAVE
BRASIL;
IESB;
BIRDLIFE

INTERNATIONAL. Complexo de Serras das Lontras e Una, Bahia: Elementos naturais e aspectos de sua conservação. São Paulo: SAVE Brasil, 2009. $60 \mathrm{f}$.

SOLLBERG, I.; SCHIAVETTI, A.; MORAES, M. E. Manejo Agrícola no Refúgio de Vida Silvestre de Una: agroflorestas como uma perspectiva de conservação. Revista Árvore, v. 38, n. 2, p. 241250, 2014. https://doi.org/10.1590/S010067622014000200004

TORRES, F. T. P. Análise do perfil dos incêndios florestais no Parque Estadual da Serra do Brigadeiro e entorno (MG). Ciência Florestal, v. $28, \quad$ n. $3, \quad$ p. 1008-1021, 2018. http://dx.doi.org/10.5902/1980509833384

TORRES, F. T. P. Risk mapping of fires in vegetation in the Serra do Brigadeiro State Park (MG) and surroundings. Revista Árvore v.41, n.2, p. 1-9, 2017. https://doi.org/10.1590/180690882017000200009

TRIMBLE. eCognition Developer: user guide. Munich, Germany: Trimble Germany, Document version 9.0.1, 2014. 262 f. Disponível em: <www.eCognition.com>. Acesso em: 26 de fevereiro de 2018.

USGS. United States Geological Survey 2018. Disponível em: <https://landsat.usgs.gov/whatare-band-designations-landsat-satellites $>$.

Acesso: em 22 de fevereiro de 2018

VERMOTE, E., et al. Preliminary analysis of the performance of the Landsat 8/OLI land surface reflectance product. Remote Sensing of Environment, v. 185, nov. p. 46-56, 2016. https://doi.org/10.1016/j.rse.2016.04.008

WILSON, E. H.; SADER, S. A. Detection of forest harvest type using multiple dates of Landsat TM imagery, Remote Sensing of Environment, v. $80, \quad$ p. $385-396,2002$. https://doi.org/10.1016/S0034-4257(01)00318-2

WONG, T. H., et al. (2003) Feature extraction based on object-oriented analysis. In: Proceedings of ATC 2003 Conference, Malaysia, 2003. Disponível em: <https://www.semanticscholar.org/paper/FEAT URE-EXTRACTION-BASED-ON-OBJECTORIENTED-WongT.

Mansor/b872d775d32c41741ee543149b983d67af af25bb>. Acesso em: 25 setembro de 2018.

ZANOTTA, D. C. et al. Deteç̧ão de queimadas no Pantanal a partir de classificação orientada a objeto e informações multiespectrais de sensoriamento remoto. In: Simpósio de Geotecnologias no Pantanal, 3. Anais... Cáceres, p. 800-808, 2010. 